\title{
Puente extradosado sobre el río Barrow. Diseño y análisis estructural del proceso constructivo, control geométrico y asistencia técnica durante la construcción
}

\section{Extrados Bridge Over the River Barrow. Design and Analysis of the Construction Process, Erection Engineering and Design Support During Construction}

\author{
Miguel Ángel Astiz Suárez ${ }^{\mathrm{a}}$, Marcos Sánchez Sánchez ${ }^{\mathrm{b}}$, Lucía Blanco Martínc, \\ Guillermo Ayuso Calle, Cian Long ${ }^{\mathrm{e}}$, Alfonso Ramírez Marchena ${ }^{\mathrm{f}}$ \\ ${ }^{a}$ Dr. Ingeniero de Caminos, Canales y Puertos, Carlos Fernández Casado S. L. \& U.P.M. \\ ${ }^{b}$ Ingeniero de Caminos, Canales y Puertos, ARUP \\ 'Ingeniera de Caminos, Canales y Puertos, Carlos Fernández Casado S. L. \\ ${ }^{d}$ Ingeniero de Caminos, Canales y Puertos, Carlos Fernández Casado S. L. \\ ${ }^{e}$ Civil Engineer, ARUP \\ ${ }^{f}$ Arquitecto Técnico, ARUP
}

Recibido el 16 de junio de 2019; aceptado el 21 de mayo de 2020

\begin{abstract}
RESUMEN
Este artículo se centra en los elementos constructivos del puente extradosado Rose Fitzgerald Kennedy sobre el río Barrow, de $887 \mathrm{~m}$ de longitud total y 9 vanos, con luces en los vanos principales de $230 \mathrm{~m}$ y tres torres atirantadas. El puente cruza el río en un punto en el que el ancho de este es superior a los $350 \mathrm{~m}$. Por esta razón la pila central (P4) se encuentra situada $50 \mathrm{~m}$ en el interior del río sobre la rivera este del mismo, en la zona de carrera de marea. El tablero se construyó utilizando una combinación de métodos constructivos diferentes para los vanos de aproximación, realizados mediante cimbra portante al suelo con torres intermedias y con múltiples fases para la sección transversal y los dos vanos centrales mediante carros de avance en voladizo de sección completa. La longitud de los semivoladizos era diferente dada la configuración de luces del puente y la diferencia de altura de las torres laterales frente a la torre central lo que requirió un elemento de bloqueo especialmente diseñado para las fases de cierre.
\end{abstract}

(C)2022 Hormigón y Acero, la revista de la Asociación Española de Ingeniería Estructural (ACHE). Publicado por Cinter Divulgación Técnica S.L. Este es un artículo de acceso abierto distribuido bajo los términos de la licencia de uso Creative Commons (CC BY-NC-ND 4.0)

PALABRAS CLAVE: Extradorsal, postesado, hormigón, gran luz, multi-atirantado.

\section{ABSTRACT}

Este artículo se centra en los elementos constructivos del puente extradosado Rose Fitzgerald Kennedy sobre el río Barrow, de $887 \mathrm{~m}$ de longitud total y 9 vanos, con luces en los vanos principales de $230 \mathrm{~m}$ y tres torres atirantadas. El puente cruza el río en un punto en el que el ancho de este es superior a los $350 \mathrm{~m}$. Por esta razón la pila central (P4) se encuentra situada $50 \mathrm{~m}$ en el interior del río sobre la rivera este del mismo, en la zona de carrera de marea. El tablero se construyó utilizando una combinación de métodos constructivos diferentes para los vanos de aproximación, realizados mediante cimbra portante al suelo con torres intermedias y con múltiples fases para la sección transversal y los dos vanos centrales mediante carros de avance en voladizo de sección completa. La longitud de los semivoladizos era diferente dada la configuración de luces del puente y la diferencia de altura de las torres laterales frente a la torre central lo que requirió un elemento de bloqueo especialmente diseñado para las fases de cierre.

@2022 Hormigón y Acero, the journal of the Spanish Association of Structural Engineering (ACHE). Published by Cinter Divulgación Técnica S.L. This is an open-access article distributed under the terms of the Creative Commons (CC BY-NC-ND 4.0) License

KEYWORDS: Extrados, posttensioning, multi-span cable supported, long span.

* Persona de contacto / Corresponding author:

Correo-e / e-mail: marcos.sanchez@arup.com (Marcos Sánchez Sánchez). 
1.

\section{INTRODUCCIÓN Y PRINCIPALES CONDICIONANTES}

\subsection{Descripción general de la estructura}

El puente sobre el río Barrow tiene una longitud total de $887 \mathrm{~m}$ con una distribución de vanos de 36-45-95-230-23095-70-50-36 m. Tres de los apoyos presentan torres de atirantamiento extradorsal de diferente altura, siendo más alta la central $(27 \mathrm{~m})$ que las laterales $(16.2 \mathrm{~m})$, las torres tienen un número diferente de cables dispuestos en arpa en un plano central ( 8 en las laterales y 18 en la central) pasantes con un sistema de silla sobre el pilono.

El tablero tiene un canto de $3.5 \mathrm{~m}$ que se incrementa linealmente en las pilas 3 y 5 a $6.5 \mathrm{~m}$ y en pila 4 a $8.5 \mathrm{~m}$ mediante una cartela lineal, asimismo tiene un ancho variable entre 19.9 y $22.50 \mathrm{~m}$ y, por exigencias de contrato, una apariencia cerrada con un alma inclinada exterior y un voladizo reducido.

\subsection{Principales condicionantes}

El puente cuenta como principal obstáculo el cruce del propio río, que tiene en la zona de cruce un ancho aproximado de $350 \mathrm{~m}$ y está situado en una zona con carrea de marea de más de $3 \mathrm{~m}$.

La posición y altura de las pilas y pilonos estaba fuertemente restringida por contrato, así como la necesidad de mantener el canal de navegación de 117 m de luz y $36 \mathrm{~m}$ de altura en uno de los dos vanos principales entre las pilas 3 y 4 .

Por razones medioambientales, el área máxima a ocupar en el río teniendo en cuenta su carrera de marea estaba limitada tanto en construcción como en la situación definitiva.

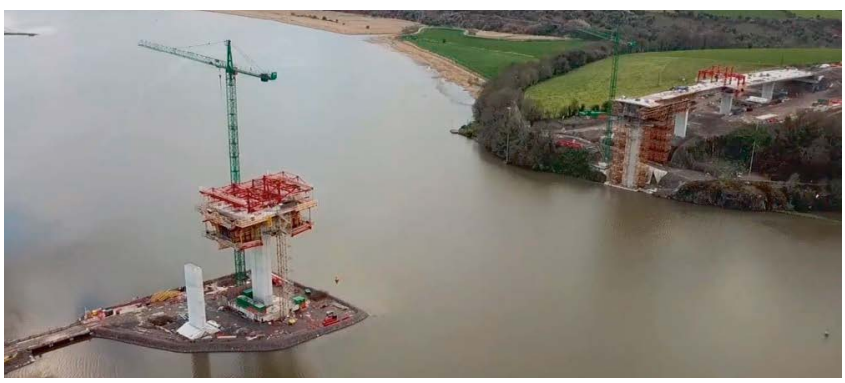

Figura 1. Cruce del rio durante la construcción (P4 central -derechay P3 lateral -izquierda-).

\subsection{Elementos generales del proceso constructivo}

Tras un estudio detallado de optimización de la solución, orientado principalmente a simplificar el proceso constructivo y reducir los plazos, se decidió ejecutar la estructura con dife- rentes métodos constructivos para los viaductos de aproximación frente a los vanos principales.

Como se describe en la sección 3, desde el punto de vista de la sección transversal, la solución fue diferente para la zona cimbrada (sección por fases) que para los carros de avance (sección completa).

\section{2.}

\section{CIMENTACIONES Y SUBESTRUCTURA}

\subsection{Estribo 1 a pila 2}

Las pilas del lado oeste de la estructura (estribo 1 a pila 3) encuentran material portante en capas someras por lo que se pudieron realizar "in situ" con excavaciones locales.

\subsection{Pila 3}

La pila 3, que se encuentra junto al río, encajonada en planta entre una carretera local existente (Pink rock road) y la margen del río en una zona de talud natural casi vertical en roca, se ha cimentado en una cota de roca ligeramente por debajo de la carrera de marea. Dado el talud vertical requerido para el acceso a la cimentación y la necesidad de reponer la carretera en su situación final, fue necesario ejecutar un muro auxiliar tras la pila.

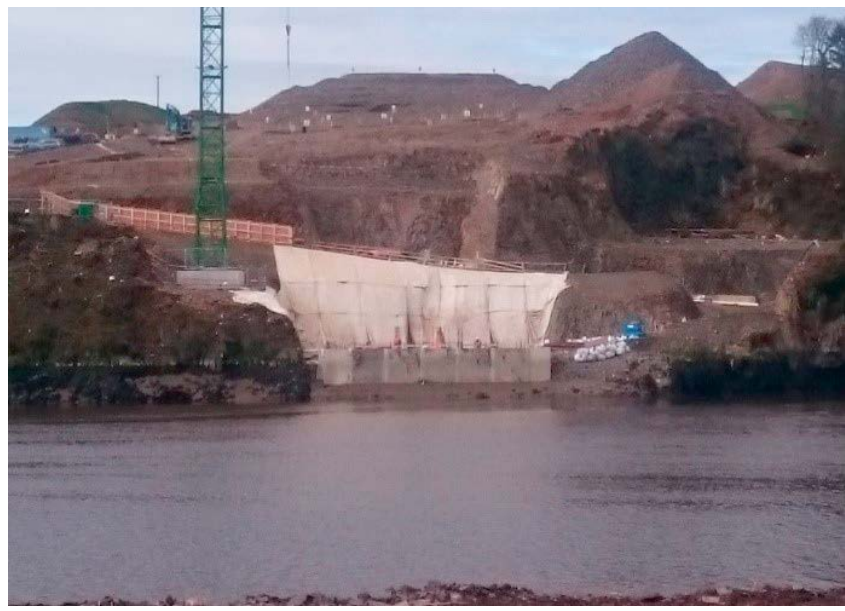

Figura 3. Cruce del río durante la construcción (P4 central -derechay P3 lateral -izquierda-).

Al encontrarse la cimentación de la pila por debajo de la carrera de marea, fue necesario ejecutar una protección provisional durante la construcción.

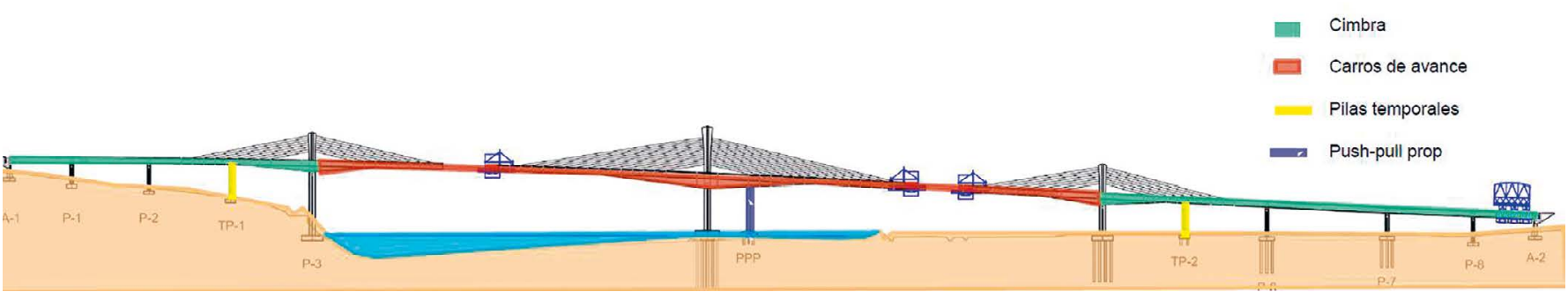

Figura 2. Esquema general del proceso constructivo del tablero. 


\subsection{Pila 4}

La pila central, que recibe cargas verticales muy importantes, requiere un encepado pilotado formado por 42 pilotes de $1.20 \mathrm{~m}$ de diámetro cuya forma se optimizó tanto en planta como en altura mediante la ejecución de un plinto, limitando el canto a $2.8 \mathrm{~m}$ más $2.6 \mathrm{~m}$ en la zona del plinto.

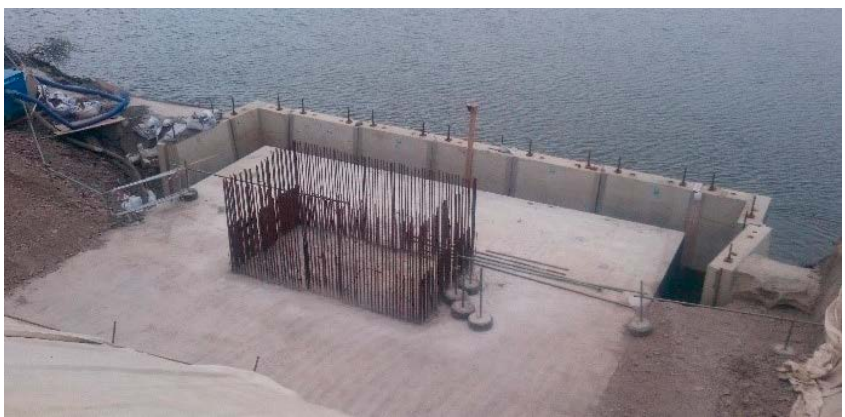

Figura 4. Cruce del río durante la construcción (P4 central -derechay P3 lateral -izquierda-).

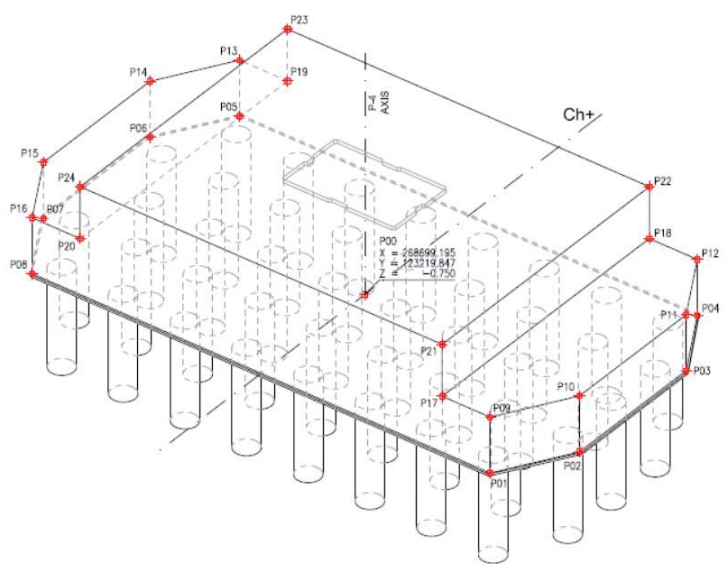

Figura 5. Encepado de la pila central (P-4).

Para la realización de este encepado y la torre provisional de equilibrio del voladizo ("PPP" o Push-Pull Prop), se realizó una península temporal con un camino de acceso que permitió la ejecución de ambas cimentaciones sin la necesidad de tablestacado, entre otras cosas debido a la posibilidad de ejecutar la cimentación con una cota inferior de $-0.75 \mathrm{~m}$, y una carrera de marea máxima a cota $4.0 \mathrm{~m}$.

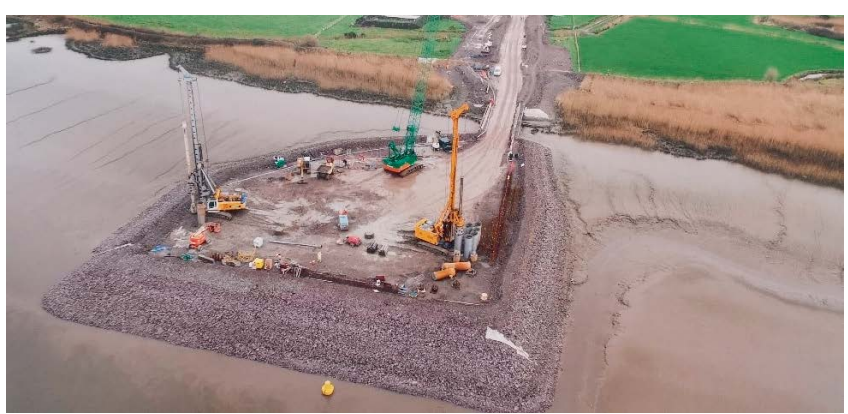

Figura 6. Península temporal para la pila central (P-4)

La decisión del uso de pilotes de pequeño diámetro $(1.2 \mathrm{~m})$ frente a una solución de pilote de mayor diámetro, más común en España se debió fundamentalmente a la ausencia de maqui- naria adecuada en la industria local para la realización de estos. Los pilotes tienen una longitud de $31.7 \mathrm{~m}$.

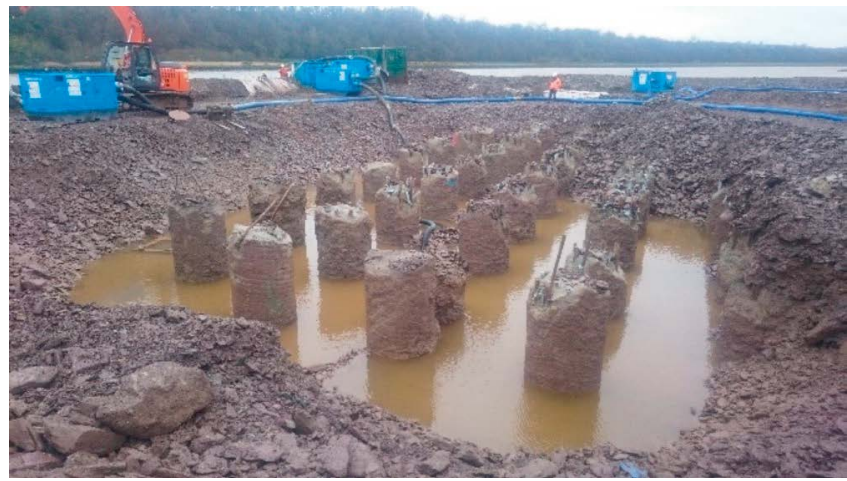

Figura 7. Pilotaje de la pila central.

Al encontrarse la cara inferior del encepado a una cota inferior a la carrera de marea alta, fue necesario ejecutar el encepado con un sistema de bombeo continuo para asegurar las condiciones adecuadas.

\subsection{Pilas 5 a 8 y estribo 2}

El resto de las cimentaciones hacia el este del puente se encuentran situadas en terreno seco fuera del cauce del río, y, en función de las diferentes condiciones del terreno, se solucionaron con cimentaciones profundas o superficiales mediante medios convencionales con excavaciones localizadas.

\section{3.}

\section{TABLERO}

\subsection{General}

Como ya se ha indicado, y se muestra esquemáticamente en la figura 2, se emplearon dos métodos constructivos diferentes para los viaductos de acceso y para los vanos principales.

\subsection{Sección transversal}

De forma general, independientemente de la posición en el puente, se decidió que las almas inclinadas serían prefabricadas. Estos elementos actúan únicamente como puntales y contribuyen al comportamiento transversal de la sección.

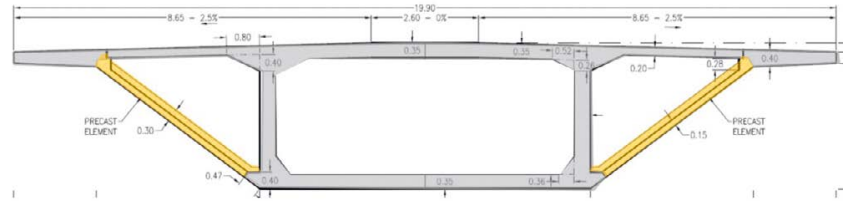

Figura 8. Sección transversal tipo.

Estos paneles, con un ancho tipo de $1.6 \mathrm{~m}$ se prefabricaron en taller y acopiaron en obra. 


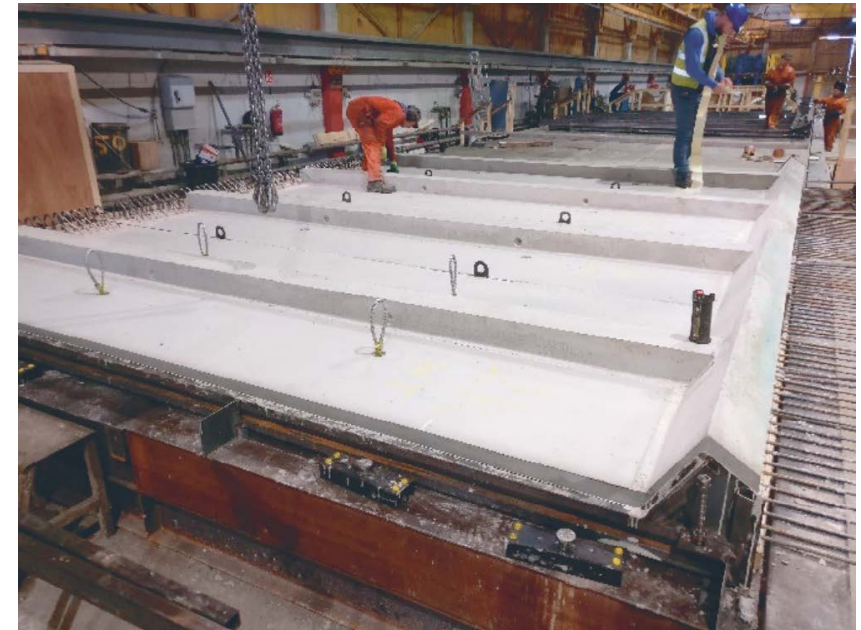

Figura 9. Panel prefabricado de alma inclinada.

\subsection{Tablero sobre cimbra}

Como ya se ha indicado, los vanos de aproximación, que comprenden del estribo 1 a pila 3 en el oeste y de pila 5 a estribo 2 en el este se realizaron mediante cimbra al terreno.

En estos tramos, la estructura se encuentra entre 5 y 20 metros sobre el terreno natural, que se encuentra dentro de los rangos habituales para este tipo de metodología constructiva.

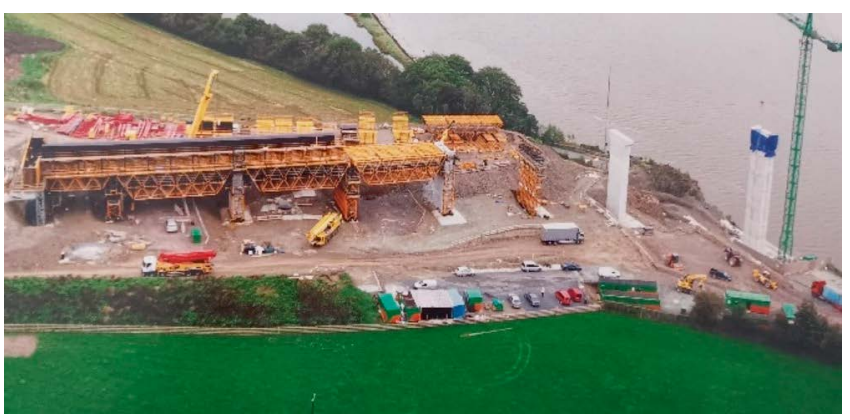

Figura 10. Tramo de cimbra sobre el vano 1 y 2.

La solución, propuesta por el equipo de construcción, se realizó buscando una minimización de los medios auxiliares y con el objetivo de acelerar el proceso constructivo y tenía las siguientes características.

- La cimbra debería tener un número reducido de apoyos al terreno para evitar ejecutar cimentaciones temporales.

- La cimbra tendría capacidad portante reducida, requiriéndose que la sección transversal se ejecutara en 3 fases (cajón $\mathrm{U}$ inferior, losa superior y alas), transfiriendo parte de la carga de las nuevas fases a las ya ejecutadas tras efectuar un pretensado parcial.

- Longitudinalmente se efectuarían tramos de la mayor longitud posible para las fases 1 y 2 , que gobernaban el trazado de pretensado de estos vanos y la disposición de diafragmas y anclajes, efectuándose la tercera fase con un carro de alas específicamente diseñado.

Debido a los requerimientos del anejo nacional del eurocódigo de la normativa irlandesa, que requiere descompresión en toda la sección [1] y no únicamente a una distancia de la vaina, esta ejecución requiere aumentar de forma significativa las cuantías de pretensado de estos vanos debido a los estados tensionales generados por la construcción multifase tanto en el cálculo global como a nivel seccional

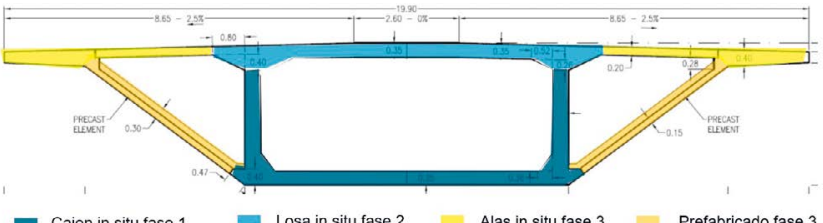

Figura 11. Fases de ejecución en cimbra.

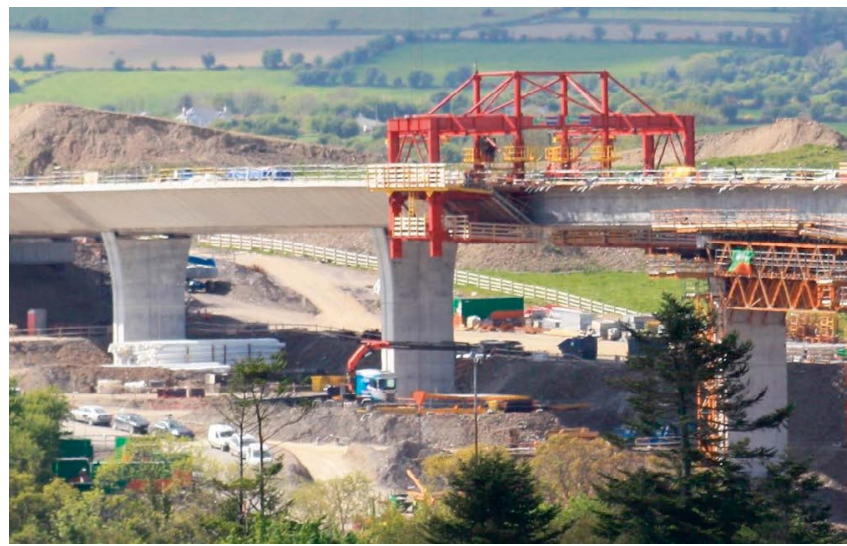

Figura 12. Carro de alas sobre vano 3.

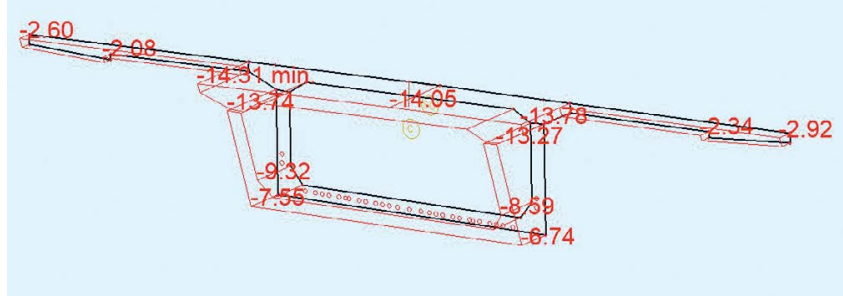

Figura 13. Sección en vano 7 bajo cargas permanentes.

\subsection{Tablero en voladizo}

\subsubsection{General}

Los vanos centrales de 230 metros de luz se efectuaron con carros de avance en voladizo con carro de sección completa.

Con el fin de minimizar el plazo se utilizaron cuatro carros idénticos. Dada la asimetría de pilonos, únicamente el pilono 4 requería avance en voladizo con dos frentes mientras que los pilonos 3 y 5 tenían un único frente al haberse realizado los vanos laterales con cimbra.

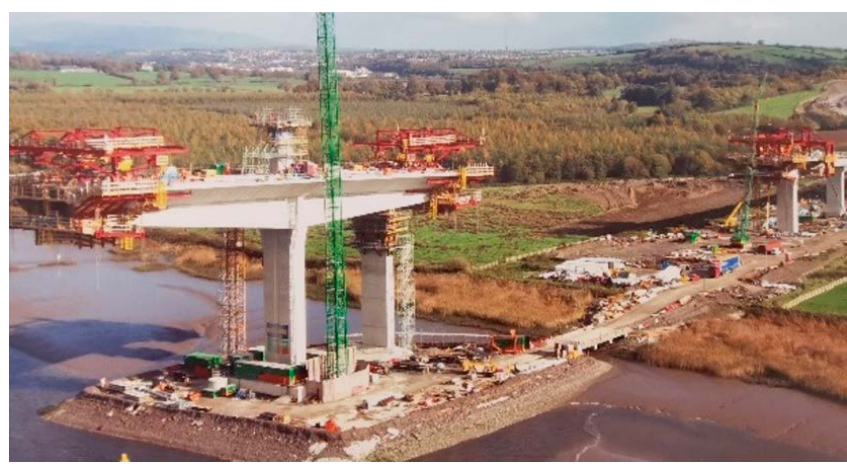

Figura 14. Voladizo en pila 4 (y Push-pull prop). 
Fue necesario disponer de 3 pilas temporales:

- Dos en los vanos laterales de $95 \mathrm{~m}$ que, al haberse ejecutado con cimbra no son capaces de resistir eficientemente su peso propio hasta que se coloquen los cables de atirantamiento, lo cual estaba condicionado al avance en voladizo desde las pilas 3 y 5. Precisamente por esta circunstancia es necesario disponer 4 tendones de pretensado exterior en los vanos laterales de $95 \mathrm{~m}$. Estos tendones se destesan una vez el tablero es continuo de estribo a estribo.

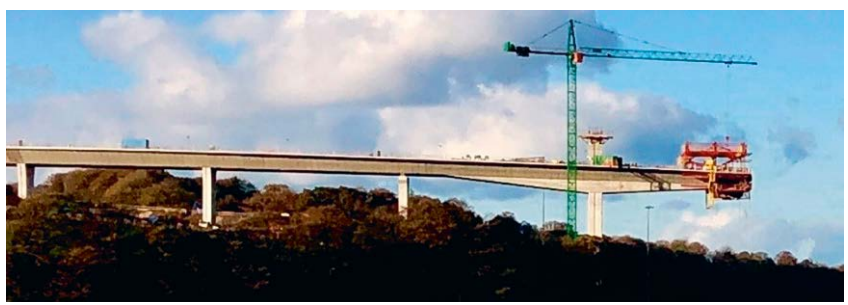

Figura 15. Pilas 1, 2, Temporal 1 y Pila 3.

- Un apoyo temporal capaz de resistir tracciones y compresiones para recoger las cargas descompensadas durante la construcción del voladizo de pila 4 .

\subsubsection{Longitud y ciclo de dovela.}

Debido al ángulo extremadamente pequeño de los cables con el tablero (entre 9 y 11 grados), la longitud de la dovela estaba condicionada por la propia separación de los cables, que se estableció en 6.50 metros.

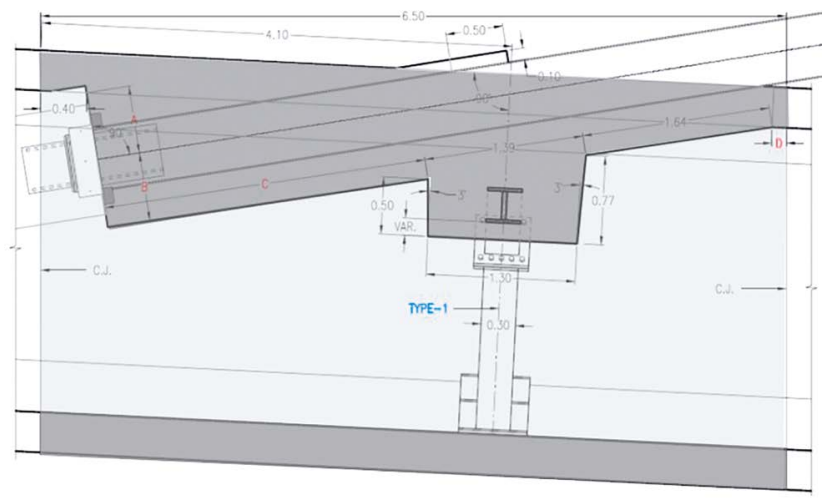

Figura 16. Sección longitudinal de la dovela tipo en con cable.

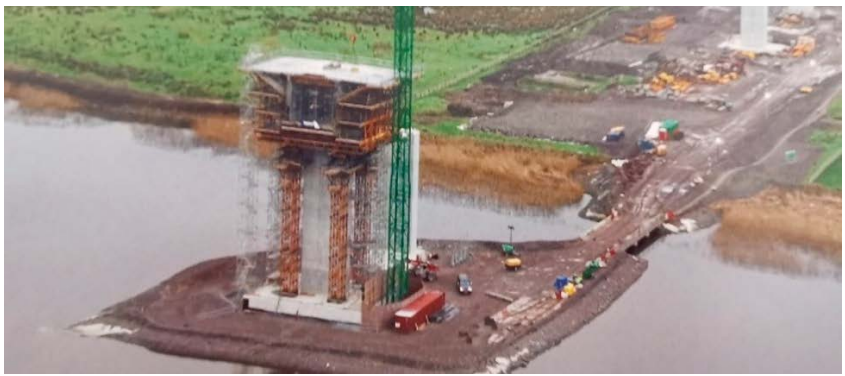

Figura 17. Dovela "0" sobre pila 4. Construída sobre cimbra.

La primera dovela sobre pila 4 se proyectó con una longitud de 12 metros para poder ejecutar la colocación de los carros. Esta dovela era además integral con la pila pues es el punto fijo del tablero respecto a movimientos horizontales en la situación de servicio.
Las primeras dovelas más cercanas a las pilas, tanto por el aumento del canto como por consiguiente el peso, como por la ausencia de cables en esta zona, se realizaron de menor longitud (4.50 metros) por capacidad de carro.

Desde el punto de vista del ciclo constructivo, la secuencia acordada con el equipo de construcción, y que condicionaba el pretensado de continuidad fue el siguiente:

- Hormigonado de la dovela "n"

- Tras un mínimo 36h, y si el hormigón alcanzaba la resistencia requerida $\left(37 \mathrm{~N} / \mathrm{mm}^{2}\right.$ en hormigones C50/60 y $50 \mathrm{~N} / \mathrm{mm} 2$ en C80/95), se realizaba el pretensado de la dovela " $n$ ".

- Avance de carro a la dovela " $n+1$ ".

- Pretensado transversal de la dovela "n-1".

- Colocación parcial de ferralla y puntales de la dovela "n+1".

- Tesado del cable principal de la dovela "n-l".

- Colocación de placas prefabricadas y resto de ferralla de dovela " $n+1$ ".

- Hormigonado de la dovela " $n+1$ ".

El pretensado longitudinal de fase, se realizó mediante cables en las primeras dovelas y, una vez el cálculo lo permitía, mediante barras pretensadas de $47 \mathrm{~mm}$ para agilizar el plazo.

Si bien en principio se previó un ciclo de hormigonado de entre 7 y 15 días, los plazos realizados en obra resultaron mucho más variables por múltiples razones, lo que complicó el control geométrico.

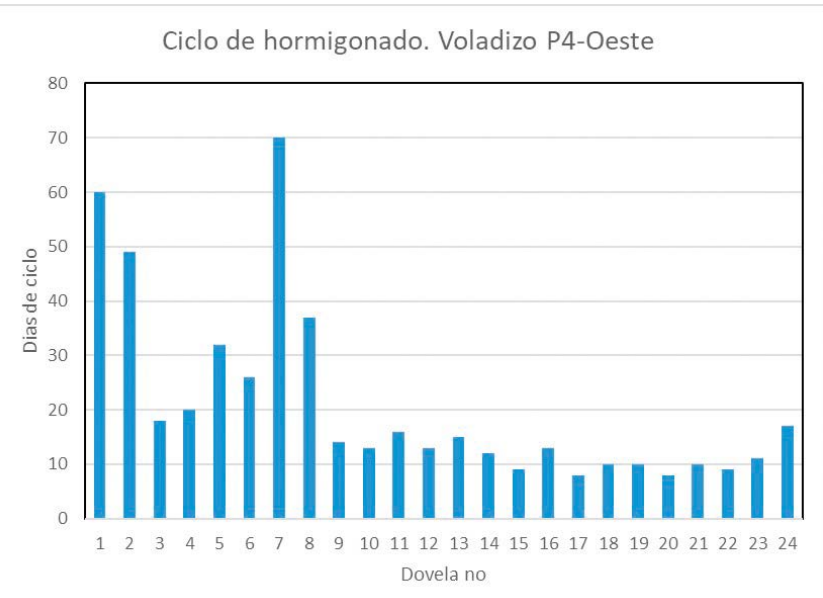

Figura 18. Tiempos de ejecución de dovelas.

Los cables de atirantamiento principal, con un número de torones variable entre 109 (pilas 3 y 5) y 109-123-125 (pila 4) se tesaron durante la construcción del voladizo a una fuerza del orden de 0.4 GUTS con el fin de optimizar las cuantías de pretensado longitudinal.

\subsubsection{Estudios específicos de fluencia y retracción}

Debido al uso de múltiples hormigones en el tablero (C50/60, C60/75 y C80/95), incluyendo hormigones de alta resistencia, se realizaron ensayos específicos de fluencia y retracción a las edades más tempranas posibles, que debido a problemas logísticos de acceso a laboratorio, fueron de 7 días.

Desde el punto de vista del análisis del proceso constructivo, se realizaron dos modelos de cálculo de fase a fase con 
dos paquetes de cálculo independientes (Sofistik- ARUP- y software propio -CFCSL-). La fluencia se implementó de forma diferente en los modelos con el fin de evaluar la influencia en las flechas previstas en la orientación de carro. En uno de los modelos se implementó la formulación de la EHE [3] mientras que en el otro modelo se realizaron los ajustes de funciones permitidos por el Eurocódigo [2], llegándose a la conclusión de que la influencia era poco significativa.

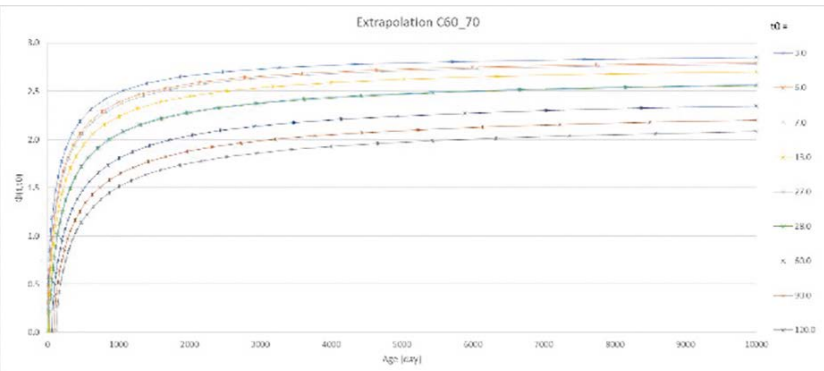

Figura 19. Curvas de fluencia basadas en el ajuste de los ensayos según Apéndice B de EN1992-2(B-104).

Para el cálculo de contraflechas, se realizó un reajuste en cada fase de construcción a los tiempos de ejecución ya realizados y previstos para el resto de la construcción. Desde un punto de vista general, la cota de cierre prevista se encontraba por debajo de la cota final ya que las fases posteriores (resto de carga permanente, retesado de cable y fluencia) producían una deformada positiva en el tablero.

\subsubsection{Comportamiento durante la construcción}

Como parte del control geométrico, se realizó una orientación de carros, contrastada contra los dos modelos realizados de forma independiente una vez por ciclo tras el hormigonado de cada dovela.

Si bien los resultados obtenidos en las primeras dovelas se encontraban dentro de las tolerancias previstas, a partir de la dovela 12 de la pila central se empezaron a encontrar discrepancias significativas entre las deformaciones previstas en los dos modelos y los resultados suministrados por topografía; esto llevó, particularmente en las dovelas 14 a 20, a un control más exhaustivo de la geometría y las deformaciones, incluso en fases intermedias del ciclo.

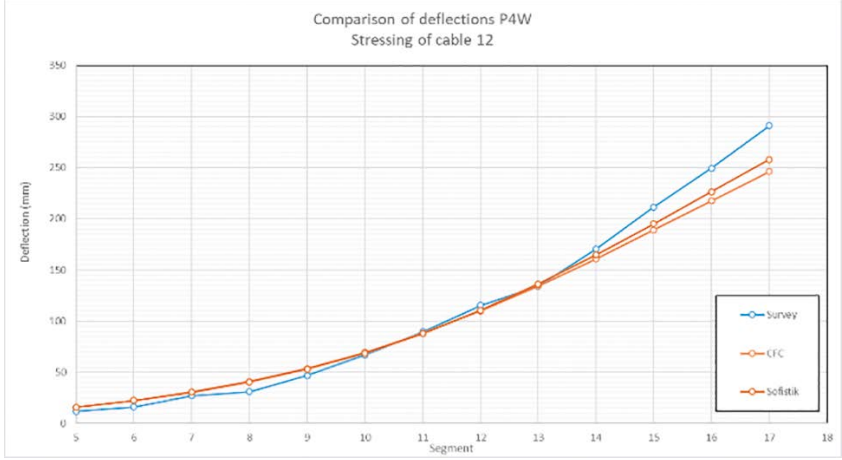

Figura 20. Comparación de deformada del voladizo oeste tras tesar el cable 12 (dovela 17).

Si bien no fue posible establecer conclusiones definitivas sobre el incremento de flexibilidad del voladizo a partir de la dovela
12, las razones más plausibles que se encontraron fueron la reducción del tiempo de ciclo y posiblemente ligeros cambios en la dosificación del hormigón de alta resistencia a partir de esta dovela, que aumentó su trabajabilidad. Como medida de prudencia se aumentó el tiempo de tesado tras hormigonado de la dovela de frente de 36 a 60 horas en el resto de las dovelas del voladizo.

\subsubsection{Elementos de bloqueo en la fase de cierre}

Debido a la diferencia en longitud de los voladizos de los vanos principales (90 metros desde las torres laterales y 140 metros desde la central) las deformaciones de los voladizos, tanto durante la construcción como durante el hormigonado de cierre son claramente diferentes.

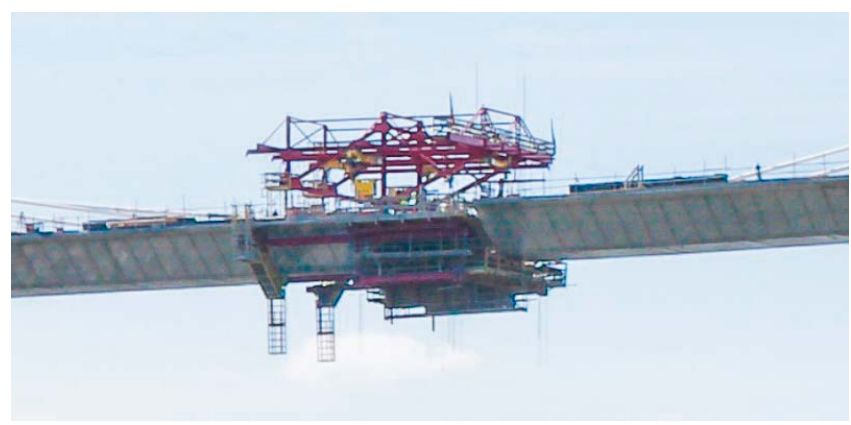

Figura 21. Flecha diferencial en voladizo de vano 4.

La mayor diferencia de flechas entre voladizos, antes de tesar el cable 17 en los voladizos de pila 4, era superior a los $700 \mathrm{~mm}$.

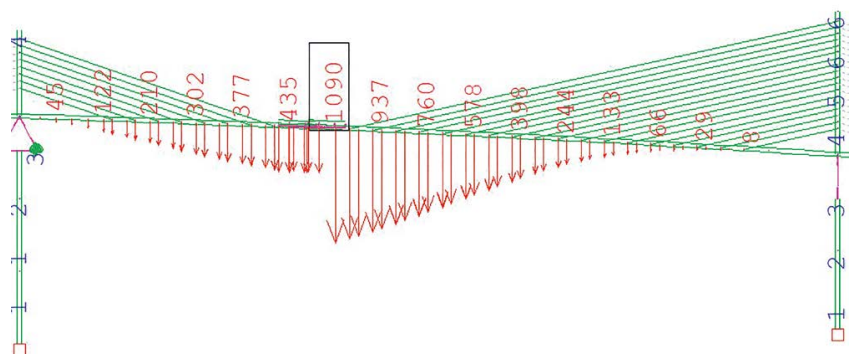

Figura 22. Flecha diferencial en voladizo de vano 4 (modelo Sofistik).

Con el fin de corregir la flecha diferencial entre voladizos, así como para asegurar que, durante las fases de cierre y hormigonado de la dovela de continuidad, no se produjeran movimientos relativos entre los dos voladizos, se diseñó un elemento específico para estas actividades. Este elemento tenía la capacidad de soportar tanto cortantes como giros.

El elemento de bloqueo consistió en un par de vigas de $1.5 \mathrm{~m}$ de canto con 4 filas de barras McAlloy que permitían no solo corregir hasta un máximo de $400 \mathrm{~mm}$ de flecha (en el cierre hubo que corregir, en línea con la previsión de los modelos, aproximadamente 120 a $180 \mathrm{~mm}$ según el voladizo) sino también asegurar que tanto los giros como los desplazamientos estaban bloqueados durante el hormigonado de la dovela de cierre.

Es importante resaltar que, durante las fases de voladizo de la pila central, los voladizos de pila 4 tenían una longitud de $140 \mathrm{~m}$, lo que teniendo en cuenta que la sección en la zona de canto constante tiene $3.50 \mathrm{~m}$, representa una gran esbeltez. 


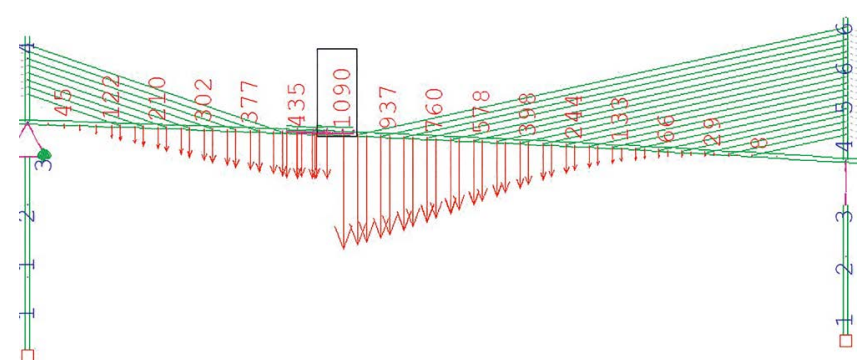

Figura 22. Vigas de bloqueo del voladizo.

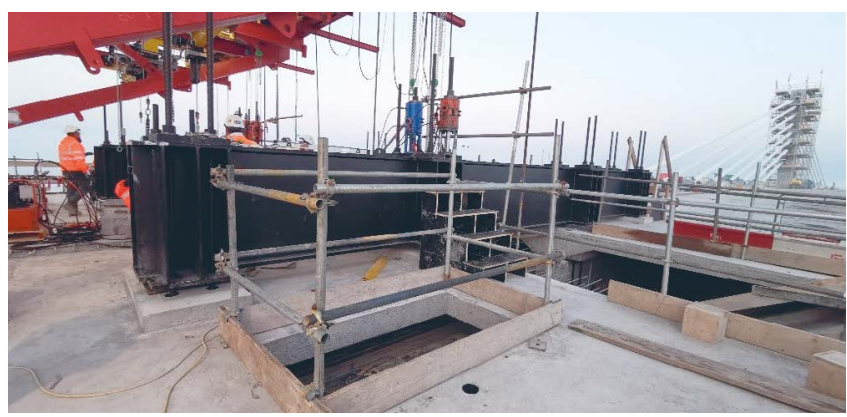

Figura 23. Alzado del puente durante el hormigonado de la última dovela de pila 4 .

\section{4.}

\section{CAMBIO DE APOYO EN PILA 5}

Tras el tesado de las vigas de cierre que proporcionaba por primera vez continuidad longitudinal a la estructura en toda su extensión, se procedió a liberar los puntos fijos horizontales, que hasta ese momento se encontraban en el estribo 1 para el tramo oeste y en la pila 5 para el este, pues el puente en su situación final se encuentra sobre apoyos "pot-teflon" guiados longitudinalmente, con la excepción de la pila central que, como ya se ha indicado, es integral con el tablero.

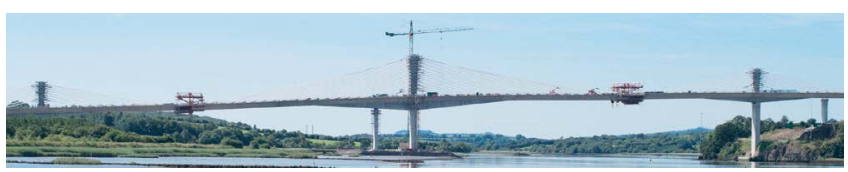

Figura 24. Apoyo tipo pot de pila 5 sobre plinto defectuoso.

En ese momento, se detectó que en la cara oculta del plinto de tablero de uno de los apoyos de pila 5, localizado bajo uno de los pilonos cortos, un defecto no registrado en el hormigón había causado una abolladura en el apoyo que obligó a efectuar una operación de cambio urgente de apoyos y reparación de la zona dañada, con dificultades añadidas de incertidumbres en otras áreas de la calidad del hormigón en la cara inferior del tablero en ese apoyo. En el momento de la detección del defecto los dos apoyos de pila 5 tenían una carga vertical de aproximadamente 4000 toneladas cada uno.

El gateado y reparación del apoyo se realizó en dos fases con el fin de minimizar el impacto en el programa de trabajos ya que el tiempo de fabricación del nuevo apoyo estaba previsto en varios meses. Para ello se utilizaron de forma tempo- ral apoyos de neopreno que habían estado dispuestos en las pilas temporales.

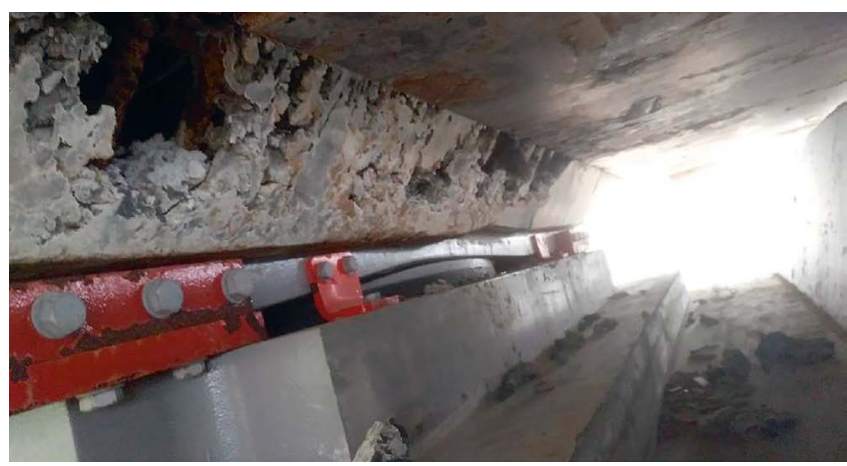

Figura 25. Sistema de gateo para la reparación y reemplazo del apoyo dañado en pila 5

5.

\section{RETESADO DE CABLES Y ACABADOS}

Por último, una vez completadas las secciones estructurales del tablero tras ejecutar las dovelas de cierre y después de ejecutar el pretensado de continuidad en la losa inferior, se diseñó un retesado de cargas de los cables principales hasta su carga final en paralelo con las actividades de colocación de carga permanente mediante un sistema de monitoreo diario y comprobación de las cargas en los cables antes y después del retesado de cada una de las 34 parejas de cables.

El puente fue finalmente abierto al tráfico en enero de 2020.

\section{6.}

\section{CONCLUSIONES}

El Puente extradosado sobre el río Barrow es un hito en el diseño y construcción de puentes de esta tipología. Siendo probablemente el de mayor luz con una sección de hormigón, su diseño y construcción ha representado un reto de importantes dimensiones para el equipo de diseño y construcción.

Dada la esbeltez del tablero, $3.5 \mathrm{~m}$ de canto con un voladizo máximo de $140 \mathrm{~m}$ en construcción y unos cables extremadamente bajos (10 grados con el tablero) el control geométrico de flechas durante la construcción fue especialmente complicado, con las dificultades añadidas del comportamiento a edades tempranas de hormigones de alta resistencia.

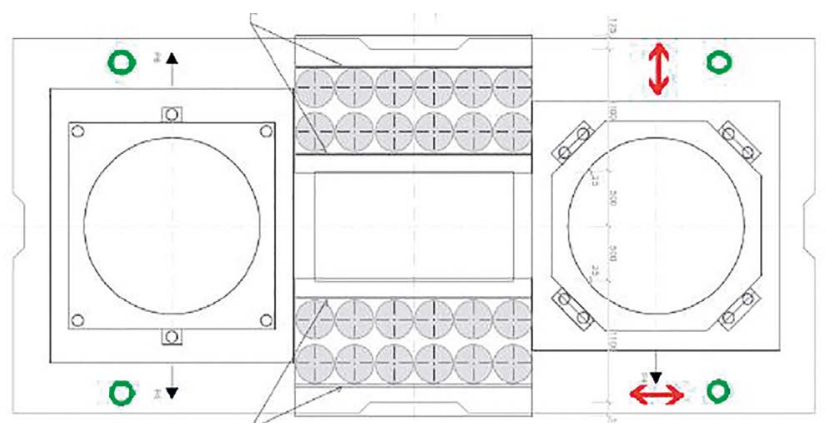

Figura 25. Sistema de gateo para la reparación y reemplazo del apoyo dañado en pila 5. 
7.

\section{AGRADECIMIENTOS}

Los autores desean mostrar su agradecimiento a otros miembros del equipo de diseño cuya colaboración ha sido fundamental en el desarrollo del proyecto, principalmente Borja Martín en CFC y Claudia Sanromán en ARUP, asimismo es importante resaltar la labor la UTE de construcción (New Ross Joint Venture) constituida por las empresas Dragados y BAM Ireland, con especial mención a los servicios Técnicos de Dragados (Ggravity) y a Pondio Ingenieros por su colaboración en los elementos de cierre y gateo del cambio de apoyo en pila 5 .
Por último a la labor realizada por los representantes de la Autoridad (Transport Infrastructure Ireland) especialmente a Mary Bowe, John Iliff y Fergal Cahill por su colaboración en el desarrollo de este proyecto.

\section{Referencias}

[1] EN 1992-1-1. Tabla 7.101 Anejo Nacional irlandés.

[2] EN 1992-2. Anejo B. Curvas de fluencia obtenidas por medios experimentales.

[3] EHE-08. Instrucción del hormigón estructural (RD 1247/2008). 